\title{
Vitamin D supplementation for the prevention or depletion of side effects of therapy with alemtuzumab in multiple sclerosis
}

This article was published in the following Dove Press journal:

Therapeutics and Clinical Risk Management

\author{
Hans-Klaus Goischke (DD \\ Independent Research, Internal Medicine, \\ Rehabilitation Medicine, Social Medicine, \\ Bad Brückenau, Bavaria, Germany
}

\begin{abstract}
Purpose of review: Not only the multiple sclerosis specialist but also the general neurologist and primary care practitioner are increasingly aware of possible adverse events (AEs) by treatment with alemtuzumab (over $47 \%$ risk of secondary autoimmune-mediated diseases). Vitamin D supplementation's effect (VDS) to reduce these autoimmune AEs is poorly performed in routine practice. This article seeks to justify why this simple, inexpensive, patient-friendly therapy should be seriously discussed.

Recent findings: Patients who have developed autoimmunity also show a high basal level of IL-21, a cytokine which increases the growth of auto-reactive T-cells. For side effects such as thyroid dysfunction, autoimmune thrombocytopenia, autoimmune hemolytic anemia, autoimmune hepatitis, diabetes mellitus type 1 , and alopecia areata/alopecia totalis, VDS may have an impact on the immunological mechanism, in particular lowering levels of IL-17 and IL-21.

Summary: The potential role of vitamin D in influencing autoimmune diseases is evident. If a life-threatening side-effect can be prevented by high-dose VDS, it is ethical to initiate this add-on therapy despite contradictory results in studies on the effectiveness of VDS.
\end{abstract}

Keywords: alemtuzumab, adverse events, hemolytic and endocrine diseases, autoimmune hepatitis, vitamin D supplementation

\section{Introduction}

Alemtuzumab (Al) is a humanized monoclonal antibody anti-CD52 that effectively depletes lymphocytes and has been approved for the treatment of adult patients with relapsing-remitting multiple sclerosis (RRMS) with active disease and is approved in more than 70 countries. Al acts by targeting CD52, an antigen primarily expressed on $\mathrm{T}$ and $\mathrm{B}$ lymphocytes, resulting in their depletion and subsequent repopulation. MS is an inflammatory condition, which is believed to be autoimmune in nature. Treatment with $\mathrm{Al}$ has been shown to increase the risk of secondary autoimmune-mediated conditions, particularly thyroid disorders (mainly AI-induced Graves' disease [GD]), including Al-associated (secondary) autoimmune thrombocytopenia (AsITP), or rarely autoimmune hemolytic anemia (AIHA), autoimmune neutropenia, red cell aplasia and single cases of Goodpasture syndrome (antiglomerular basement membrane disease) as well as membranous nephropathy. An extraordinary observation in the use of $\mathrm{Al}$ in $\mathrm{MS}$ is the occurrence of acquired autoimmune disease, which is not observed in other conditions treated with this drug - this indicates a disease-specific phenomenon. ${ }^{1}$
Correspondence: Hans-Klaus Goischke Internal Medicine, Rehabilitation Medicine, Social Medicine,

Hochwaldstrasse 2, Bad Brückenau

D-97769, Bavaria, Germany

Email hkem.goischke@t-online.de 
The Al therapy of RRMS requires at least 4 years of surveillance. So far, a drug prophylaxis of these serious side effects is not established in practice.

Baker et al have made a hypothesis after analyzing the $\mathrm{T}$ - and $\mathrm{B}$-cell repopulation after $\mathrm{Al}$ treatment, regarding the induction of secondary autoimmune diseases. The Bcell repopulation occurs much faster than that of the Tcells, especially the regulatory T-cells. Reconstitution of B-cells without adequate control (hyperrepopulation) by regulation of T-cells could be considered as the triggering cause of GD, Hashimoto's thyroiditis, and secondary immune thrombocytopenia (ITP). ${ }^{2}$

It is evident that vitamin D supplementation (VDS) yields multiple beneficial immunological effects. These positive effects could be used to prevent or mitigate secondary autoimmune diseases.

\section{Al-associated secondary autoimmune thrombocytopenia (AsITP)}

AsITP usually develops in $1-3.5 \%$ of patients with a latency period of 14-36 months (delayed ITP) after the first Al infusion; there are also reports for the period of 4 51 months. Mild transient decrease in platelet count during $\mathrm{Al}$ administration (5-day therapy cycle) is not associated with clinically relevant events and is likely related to the cytokine-release syndrome. Normalization of platelets occurring within 8 weeks without specific therapy has been observed. However, not always self-limiting, fully reversible or predictable thrombocytopenias have been registered. ${ }^{3-5}$ Monitoring in the following months after Al-infusion should be considered good clinical practice.

\section{Al-induced AIHA}

Al can be associated with rare but severe AIHA during MS treatment, also in combination with glomerular nephropathies. $^{6,7}$ AIHA is defined as the increased destruction of red blood cells in the presence of anti-red blood cells autoantibodies with or without complement activation. Barcellini investigated the pathogenesis of AIHA and favored a reduced Th1 and a predominant Th2-like profile in the immunopathogeneses of AIHA, in contrast to the prevalent role of CD8+ interferon (IFN)- $\gamma-$ secreting cytotoxic T-cells in organ-specific autoimmunity, such as MS. ${ }^{8}$

Several immunologic mechanisms are involved in the pathogenesis of AIHA, autoantibodies, antibody-dependent cell-mediated cytotoxicity, phagocytes, B and T lymphocytes, specific CD4+/CD25+ regulatory T-cells (Tregs), cytokines, and the complement system. Overview by ${ }^{8}$ in the occurrence of two or more autoimmune diseases and MS, the pathophysiology is extremely complex and so far unclear in all details.

\section{Polyautomimmunity in patients with MS - an immunological labyrinth}

Numerous publications have shown persons with multiple sclerosis (PwMS) to be susceptible to developing chronic autoimmune diseases alone without AI therapy, suggesting an autoimmune predisposition. Overview by ${ }^{9}$ studies demonstrated a pivotal role of homeostatic proliferation driven by IL21, arising from mature T-cells having escaped $\mathrm{Al}$ treatment and leading to a predominance of oligoclonal, highly proliferative, and chronically activated effector memory T-cells. ${ }^{10,11}$ The tendency to polyautoimmunity is demonstrated by the simultaneous occurrence of ITP and autoimmune thyroid disease following $\mathrm{Al}$ treatment in RRMS and simultaneous earlyonset severe AIHA and nephropathy. ${ }^{7,11}$ AsITP is associated with a unique form of ITP, is characterized by delayed onset, responsiveness to conventional therapies, and prolonged remission following treatment. ${ }^{12}$

\section{Vitamin D (VD) deficiency - a remarkable pathophysiological problem}

VD deficiency has been implicated in the pathophysiology of various inflammatory diseases including MS and hematologic autoimmune diseases.

Mean serum $25(\mathrm{OH}) \mathrm{D} 3(\mathrm{VD})$ levels have been determined to be significant lower in hematological disorders, such as ITP, AIHA, Evan's syndrome, and chronic idiopathic neutropenia $(\mathrm{CIN})$, than in controls. ${ }^{13}$ VD deficiency is very common in children with newly diagnosed or chronic ITP form. ${ }^{14}$

\section{Which immunological mechanisms are known in hematological diseases?}

Several autoimmune (eg, MS) and hematological disorders share a similar cytokine profile of higher IFN $\gamma-$, IL-6-, and IL-17-levels, as was observed at ITP, AIHA, Evan's syndrome, and CIN. IL-21, a cytokine promoter in differentiation of $\mathrm{T}$ helper cells (Th) 17, follicular helper T-cell and B-cell may thereby play an important role in controlling the autoimmune process in ITP. Circulating IL-21 levels are significantly higher in ITP-patients than that in healthy 
controls. ${ }^{15}$ IL-17A and IL-21 induce Th17-cells and inhibit $\mathrm{T}$ regulatory (Treg)-cells re-differentiation via Th17associated signaling pathway in ITP patients in vitro. ${ }^{16}$

More recently, Th17-cells, which are characterized by secretion of IL-17, have been identified as key effectors in the development of many autoimmune diseases (eg, MS), including AIHA. Elevated frequency of Th17-cells and increased IL-17 secretion were found closely correlated with the disease activity in AIHA patients. ${ }^{8}$ Higher levels of IL-21 have been shown to be associated with developing autoimmune disease post-AI therapy. ${ }^{17}$

\section{Which immunological mechanisms justify a VD supplementation?}

It is biologically plausible that sufficient VD levels may be important in modulating inflammatory processes. VD has numerous effects on cells within the immune system. It inhibits B-cell proliferation and blocks B-cell differentiation and immunoglobulin secretion. VD additionally suppresses T-cell proliferation and results in a shift from a Th1 to a Th2 phenotype. Furthermore, it affects T-cell maturation with a skewing away from the inflammatory Th17 phenotype and facilitates the induction of $\mathrm{T}$ regulatory cells. This leads to a decline in the production of inflammatory cytokines, such as IL-12, IL-17, and increased production of anti-inflammatory cytokines such as IL-10. ${ }^{13,18}$

VD also has effects on monocytes, macrophages and dendritic cells. It inhibits production of inflammatory cytokines, such as IL-1, IL-6, IL-8, IL-12, and TNF $\alpha .{ }^{19}$ • Especially, 1,25dihydroxyvitamin D3 $(1,25(\mathrm{OH}) 2 \mathrm{D} 3)$ has potent immunomodulatory properties that have promoted its potential in the prevention and treatment of autoimmune disease. ${ }^{20}$ Jeffery et al observed that stimulation of CD4+CD25-T cells in the presence of 1,25(OH)2D3 inhibited production of pro-inflammatory cytokines and stimulated expression of high levels of cytotoxic Tlymphocyte antigen 4 as well as FoxP3. It is now established that FoxP3 + regulatory T-cells are critical to the prevention of autoimmunity. The maintenance of a significant population of such cells is therefore required for lifelong health. ${ }^{20}$

Overall, the majority of cells involved in hematological diseases express a high level of vitamin D receptor (VDR). This suggests that these cells may be responsive to VD treatment. $^{21}$ In ITP, AIHA and CIN VD receptor(R) expression levels were higher in the disease state compared to normal donors. $^{13}$

From a prophylactic point of view, to avoid AsITP, VDS should be carried out to an optimal 25(OH)D serum level. Benefits were demonstrated by VD administration in ITP. ${ }^{13,22,23}$ ITP also showed a lower number of platelets in cases with very low VD levels, and Liu et al also observed lower 1,25(OH)2D3 values with active ITP. $^{24}$

$1,25(\mathrm{OH}) 2 \mathrm{D} 3$ has two important immunomodulatory functions: Lower regulation of CD4+ cell overproduction combined with up-regulation of T-regulatory cells (Treg) could therefore reduce the autoantibody response and restore normal platelet levels. $1,25(\mathrm{OH}) 2 \mathrm{D} 3$ as a potent immunomodulator inhibits inter alia the production of the proinflammatory cytokines IL-17 and IL-21, which could be a potential marker for increased autoimmunity in MS, as well as increased production of anti-inflammatory cytokine IL-10. IL-10 plays an essential und highly complex role in the modulation of adaptive immune responses. IL-10 inhibits production of a number of proinflammatory cytokines, including IL-1 $\beta$, IL-6, IL-12, IL-18, granulocyte-macrophage colony-stimulating factor, and TNF $\alpha$. Overview $\mathrm{by}^{25}$ regarding the regulatory role of B-cells and IL-10 production in the resolving of autoimmune diseases, there is currently a significant increase in knowledge. The interpretation of elevated levels of IL-10 is also complicated by the reports of IL-10-independent mechanisms in autoimmune diseases. ${ }^{26}$ The pleiotropic nature of IL-10 makes it difficult to introduce the benefit of IL-10-modulating therapies into the clinic.

\section{Practical recommendations - AsITP}

All therapeutic and diagnostic options must be exploited to prevent or detect timely and rare adverse effects. However, rare and late adverse events (AEs) are often identified only during post-marketing observation. ${ }^{27}$

VD may very well be a significant factor in preventing the loss of tolerance to self and resultant polyautoimmunity. The optimal VD supplementation results in multiple beneficial immunological effects in multiple sclerosis. ${ }^{28}$. The demonstrated immunological mechanisms in hematological diseases - ITP, AIHA-demand VD supplementation, especially since the VD application has few side effects. Although VD therapy is initially hypothetical for preventing side effects from AI, its effectiveness should be confirmed to increase patient safety.

If platelet counts of $\geq 50,000 / \mu \mathrm{L}$ but $<100,000 / \mu \mathrm{L}$ (two short-term controls after a few days) are registered, immediate initiation of high-dose VD supplementation may be initiated prior to the side-effect rich first-line therapy. These platelet counts could be the putative "window of 
therapeutic opportunity" with VD being a meaningful "supportive first line option". If cutaneous signs of thrombocytopenia occur (petechiae, ecchymoses) and the platelet counts are $<50,000 / \mu \mathrm{L}$, equally high-dose VD would be indicated as "first-line co-medication". The differential diagnosis of thrombocytopenia is facilitated if Al-treated patients refrain from the consumption of quinine-containing drinks and medicines (tonic water, bitter lemon, etc.), as this substance may cause secondary (drug-related) sudden, severe thrombocytopenia. ${ }^{29}$

Furthermore, there is a correlation of VD deficiency and thyroid autoimmune diseases. Serious VD deficiency $(<10 \mathrm{ng} / \mathrm{mL})$ corresponds with Hashimoto's thyroiditis, $\mathrm{GD}$, and hypothyroidism.

\section{Considerations for clinical practice in hematological AEs}

The current state of knowledge gives no reference of a predisposition to PwMS that could lead to developing an autoimmune disease. There are no biomarkers that signal polyautoimmunity which could occur with AI therapy. The results of the studies allow a VDS without restriction. 25 $(\mathrm{OH}) \mathrm{D} 3$-serum levels are typically lower in PwMS and hematological disease compared to healthy individuals. Diseased cells typically express a high level of VDR. In abnormal hematological cells, VDS reduced the production of pro-inflammatory cytokines. ${ }^{21}$ In patients with autoimmune cytopenias, it was possible to verify these theses: 1) VD is reduced and its receptor increased in autoimmune cytopenias; 2) VD deficiency is associated with ITP and AIHA severity at onset; 3) low VD levels are associated with increased relapse rates in AIHA; and 4) VD inhibits anti-erythrocyte autoantibody production in vitro. ${ }^{13}$ For the prevention of side effects (autoimmune and hematological diseases) under therapy with Al, VDS should be used in particular for AsITP and AIHA. It could affect the severity of the disease, as it has protective and immunomodulatory effects. ${ }^{13}$ The recent case reports on AIHA in RRMS treatment with $\mathrm{AI}^{30}$ could expand the surveillance program by determining the Coomb's test. ${ }^{6,7}$ Due to the possible lifethreatening comorbidities due to an autoimmune side effect, a physician applying this treatment should be cautious. The successful implementation of an automated electronic support system for safety monitoring of multiple sclerosis patients demonstrates its importance. In one case of AsITP, while the treating neurologist was on leave, the automated analysis of the pathology results provided rapid treatment. ${ }^{31}$

\section{Quick recognition of unexpected adverse effects as prevention of life- threatening situations Secondary hemophagocytic lymphohistiocytosis}

Rare and late AEs are often identified only during postmarketing observation. In the monthly $\mathrm{Al}$ monitoring program (blood tests), the determination of C-reactive protein (CRP) in the differential diagnosis may be helpful. In the presence of anemia, thrombocytopenia, pancytopenia and elevated CRP, a rare secondary hemophagocytic lymphohistiocytosis may be the cause that has been previously reported in two cases. ${ }^{32}$ This hyperinflammatory syndrome has occurred among others in autoimmune disease and this syndrome is characterized mainly by fever, pancytopenia, hyperferritinemia, pathological liver counts, raised soluble IL-2 receptor (CD 25), lymphadenopathy, and hemophagocytosis.

\section{Acute acalculous cholecystitis (AAC)}

If acute abdominal pain occurs in the upper right quadrant shortly after Al infusion, a differential diagnosis of very acute AAC must be considered. An AAC represents a new and potential life-threatening adverse event. Croteau et al described eight cases with AAC, four cases were assessed as probable while four were possible. The clinical symptoms occurred during or shortly after $\mathrm{Al}$ infusion. Although the pathophysiological mechanism has not been fully elucidated, an "acute cytokine release syndrome" may be the cause. One patient experiences an AAC about 45 days after completion of the first cycle of AI infusion. ${ }^{33}$

\section{VDS as a further prevention measure of rare side effects of $\mathrm{Al}$ therapy Alopecia areata (AA)/alopecia totalis (AT)} Rare side effects of $\mathrm{Al}$ therapy are the occurrence of AA or $\mathrm{AT},{ }^{34,35}$ which comes with a substantial health care burden. Zimmermann et al and Leussink et al provide evidence that AA and AT is an additional, but barely recognized, secondary autoimmune disease after $\mathrm{Al}$ application. ${ }^{34,35}$ The predisposition of patients with MSfor polyautoimmunity is corroborated by these case histories. AA is a common, recurrent, autoimmune hair disorder. ${ }^{36}$ Severe forms of AA, such as AT or alopecia universalis are strongly linked to thyroid autoimmunity ranging from $25 \%$ to $40 \%$ of all cases. ${ }^{37,38}$ The complexity of pathophysiology is demonstrated by the fact that thyroid 
disorders predominantly show B-cell but also a $\mathrm{T}$ cell mediated autoimmunity. AA is an autoimmune disease resulting from $\mathrm{T}$-cell mediated (Th1 category) damage to the hair follicle. ${ }^{39} \mathrm{CD} 8+\mathrm{NKG} 2 \mathrm{D}+\mathrm{T}$ cells promote AA pathogenesis, acting as cytolytic effectors responsible for autoimmune attack of the hair follicle. IFN- $\gamma$ produced by CD8 T-cells leads to the collapse of immune privilege in the hair follicle. ${ }^{39}$ However, the lesional skin in AA also shows a mixed profile - including signs of a classic Th2 disease. ${ }^{40}$ Serum $25(\mathrm{OH}) \mathrm{D}$ levels are lower in patients with AA than healthy control samples. ${ }^{41,42}$ These levels were negatively correlated with disease severity and pattern of hair loss. This may provide evidence about the role of VD deficiency in AA pathogenesis. Severe AA showed by far the lowest VD levels compared with cases with mild or moderate disease. ${ }^{43}$ This result was suggested to be caused by its effects on the hair and the immune system. Expression of VD-receptors in keratinocytes is necessary for maintenance of the normal hair cycle. VD inhibits the secretion of proinflammatory cytokines, including IL-2 and IFN- $\gamma$. IL-2 and its receptors play a key role in the proliferation of autoreactive T-cells, but also in the loss of immune tolerance in MS. Because serum 25(OH)D levels were significantly lower in AA, VD supplementation with serum levels in the upper physiological range is also justified here. $^{41,42}$

Chan et al report on another case of AA and they could register a great therapeutic success by a "therapeutic" coincidence. ${ }^{44}$ Local therapy with triamcinolone acetonide with intralesional injections was not very successful. Because of a relapse, the female MS patient intravenous received 1,000 $\mathrm{mg}$ methylprednisolon/day over 5 days. This "therapeutic coincidence" led to a distinct improvement in AA.

\section{Al-induced thyroid dysfunction (AITD)}

Al-induced thyroid disease (thyroid AEs combined with abnormal thyroid function test) occurs in up to $40 \%$ of patients. AITD can significantly affect the quality of life of MS patients if this side effect is not recognized in time and adequately responded to by all specialist disciplines. Before AI therapy, the TSH-(thyroid-stimulating hormone) level and the thyroid gland should be examined. In the detection of TPO-(thyroid peroxidase)-AB before the start of treatment, the risk of developing an AITD was $69 \%$ compared to TPO-negative patients with only $31 \%$ risk. Eighty-five percent of patients who developed AITD later were TPO-AB-negative before therapy, meaning baseline AB-negativity is no protection from AITD. ${ }^{1}$

Daniels et al have observed 102 episodes of thyroid dysfunction in 216 AI-treated patients. Seventy-three patients (34\%) developed AITD, which manifested in $65.8 \%$ of the cohort as GD, in $20.5 \%$ as hypothyroidism (HYPOT) and in $12.3 \%$ as subacute thyroiditis (SLT). ${ }^{1}$ Of 86 patients, Tuchy et al diagnosed $41 \%$ with AITD, of which 63\% developed GD, 34\% developed HYPOT (with positive TPO AB), and one patient with transient thyroiditis. ${ }^{45}$ Cossburn et al reported a high AITD rate of $77 \%$, with 71\% developing GD, 17\% HYPOT, and 12\% developing transient thyroiditis. ${ }^{46}$

Several studies reaffirm that the GD is the most common occurring condition. ${ }^{47}$ For the practical procedure, the reports about the variants of the clinical course and the laboratory parameters of the AITD are helpful. Overt Graves' hyperthyroidism spontaneously resolved itself in $36.7 \%$ of patients, $20.4 \%$ became euthyroid, whereas $16.3 \%$ became hypothyroidic. ${ }^{1}$ The SLT with hyper, euthyroid, and hypothyroid phases develops in about $50 \%$ of patients to definitive hypothyroidism. ${ }^{48}$

Between GD and thyroiditis, there are transitional forms and one disease can transition into the other. Unexpected clinical courses that turn the GD into HYPOT both after long antithyroid treatment as well as without antithyroid drugs transformed must be accounted for within the longterm care plan. In the review by Rotondi et al, hyperthyroid patients have an unusually high rate of spontaneous shifts to a hypothyroidism. ${ }^{49}$ In a recent study, thyroid dysfunction was reported in $42 \%$ of patients, of which $72 \%$ developed GD, 12\% HYPO, 6\% Hashimoto's thyroiditis, and 5\% thyroiditis (defined as thyrotoxicosis followed by spontaneous euthyroidism or hypothyroidism, with negative TSH receptor autoantibodies (TRAbs) and/or absent tracer uptake on technetium scan). ${ }^{50}$ In contrast to previous reports, Pariani et al noted a more frequent incidence of GD requiring definitive or prolonged antithyroid drug treatment. ${ }^{50}$ The cause of unusual clinical observations and changing biochemical markers could be explained by an imbalance of TSH receptor stimulating AK (TSAbs) and TSH-blocking $\mathrm{AK}$ (TBAb) is coming. ${ }^{1}$

In general, spontaneous remissions of Graves' hyperthyroidism occur when TRAbs disappear, when TSAbs are balanced by antibodies that block thyrotropin action, or when persistent TSAbs are thwarted by associated autoimmune thyroiditis. 1,50 
Numerous case-based reports of an AITD document the colorful image with light forms and heavier gradients (especially in smokers) of different responses to the therapy and variants in the course of the disease. ${ }^{51}$ In isolated cases of thyroid $A B$ without evidence of subclinical HYPOT or HYPERT and a lack of pathological TSH levels, one check is sufficient.

\section{Relationship between VD serum levels and autoimmune thyroid dysfunction}

There is an evident correlation between VD deficiency and thyroid autoimmune disease. ${ }^{52}$

Although the results from studies are controversial and unresolved questions remain, there is evidence that a correlation exists between severe VD deficiency $(<10 \mathrm{ng} / \mathrm{mL})$ and Hashimoto's thyroiditis and GD, ${ }^{53-55}$ as well as hypothyroidism. ${ }^{56}$ Low 25(OH)D3-serum levels (20-30 $\mathrm{ng} / \mathrm{mL}=50-75 \mathrm{nmol} / \mathrm{L}$ ) were found particularly in young women with elevated $\mathrm{TG}-\mathrm{AK}^{57}$ and moderately elevated TPO-AK. ${ }^{58}$ On the other hand, low TSH levels were seen in individuals with high VD levels. ${ }^{59}$

If AITD develops in more than $40 \%$ of patients after AI therapy, using the current state of our knowledge, it should be discussed whether it would be better to initiate an add-on treatment with high-dose oral VD (eg, 4-10,000 IU/day) before the series of infusions. In addition, for the argumentation of a VD supplementation (14,000 IU/day), the success rate indicates that the number of active lesions in MRI was about 1/3 lower and the volume increase in the T2 lesions was halved. In young MS patients (under 30 years), no new $\mathrm{T} 1$ lesions were registered in $86 \%$ of cases. $^{60}$

Where it is demonstrable that over $40 \%$ of patients develop an AITD after AI therapy and VD deficiency may encourage the development of autoimmune thyroid disease, VDS should take priority in the interests of patient safety.

\section{Considerations for clinical practice in thyroid dysfunction}

Hyperthyroid patients (AI-induced GD [reconstitutions GD]) have an unusually high rate of spontaneous shift to hypothyroidism. The remission rate of Graves' hyperthyroidism, both spontaneous and after antithyroid drugs is unexpectedly high. ${ }^{49}$ However, long-term observations also show that GD is more likely to require definitive or prolonged antithyroid drug treatment. For reasons of safety, because of clinically atypical forms, it may be advisable to carry out the monitoring with annual TSH level determinations over a longer period of time. ${ }^{50}$

\section{Early neutropenia after Al infusion cycles - non-immunologically induced}

Increased attention must be given to AI-induced (early) neutropenia. The polymorphonuclear neutrophils also express CD52 and may be depleted by AI, thus potentially contributing to the infections that develop post-AI treatment. The degree of neutropenia was generally mild. Grade 3-4 neutropenia $\left(<1.0 \times 10^{9} / \mathrm{L}\right)$ occured in $<1.5 \%$ of PwMS in each AI treatment cycle. Two PwMS developed severe neutropenia-related AEs. ${ }^{61}$ Gaitán et al report two cases with early neutropenia. In one case, 4 weeks after standard AI induction, a severe neutropenia was developed with an absolute neutrophil count (ANC) of $470 / \mu \mathrm{L}$. In the second case, a neutropenia with values of $300 / \mu \mathrm{L}$ was observed in the first $\mathrm{AI}$ infusion series. In the second series of $\mathrm{AI}$ infusions, a transient neutropenia occurred after 5 weeks (ANC 398/ $\mu \mathrm{L}$ ) for 6 days. ${ }^{62}$ Galgani et al reported a case of asymptomatic leukopenia with neutropenia detected approximately 1 month after the first AI course with spontaneous resolution. ${ }^{63}$ A case of early neutropenia with fatal outcomes in a 47-yearold PwMS patient 23 days after AI infusion was described. Weekly blood tests for the first 2 months after the first infusion could prevent major infections. ${ }^{64}$ This "early neutropenia after alemtuzumab infusion" is to be distinguished from a "neutropenia as a result of alemtuzumab-induced secondary autoimmunity" which occurs much later in the context of immune reconstitution. ${ }^{65}$

\section{Diabetes mellitus type I after Al therapy}

The tendency to polyautoimmunity in PwMS is confirmed by the occurrence of autoimmune diabetes mellitus type 1 (T1DM) and autoimmune thyroid disease (GD) after AI therapy in three cases. ${ }^{66,67}$ The observation of three autoimmune diseases in PwMS is associated with hyperactivity of the adaptive immune system (T- and B-cells lymphocytes). An adequate VD status has been observed with a decreased risk for T1DM. ${ }^{68}$ VD represents a candidate protective factor for T1DM as it regulates the immune system and autoimmunity. The chance to prevent the T1DM lies in the administration of VD before the onset of AI cycles. The therapeutic window for VD supplementation lies in the period of "prevention", since the destruction of $\beta$-cells cannot be reversed.

Before diagnosis, patients with T1DM had lower 25(OH) D levels than controls. ${ }^{69}$ Patients with various autoimmune 
diseases showed a low VD level. ${ }^{70}$ The benefit of adequate VD supplementation was also demonstrated by administration of 2,000 IU/day from the first to the 31st year of age. There, children had a 78\% lower risk of developing T1DM compared to this person who did not receive this supplementation. ${ }^{71,72}$ Adults with lower serum 25(OH)D levels were at higher risk for insulin-dependent diabetes than those at higher levels. A 3.5-fold lower risk was associated with a serum of $25(\mathrm{OH}) \geq 60 \mathrm{nmol} / \mathrm{L}$. Individuals with a serum level of $25(\mathrm{OH}) \mathrm{D}>100 \mathrm{nmol} / \mathrm{L}$ had a $70 \%$ lower risk of developing insulin-dependent diabetes than subjects below $43 \mathrm{nmol} / \mathrm{L}^{73}$ There is a significant association between inadequate levels of $25(\mathrm{OH}) \mathrm{D}$ and higher levels of cytokine (eg, IFN $\gamma$, TNF- $\alpha$, IL-6, IL-1 $\beta$, etc.), high titers of ICA (islet cell antibodies), anti- GAD-AK (glutamic acid decarboxylase antibody) and IAA (anti-insulin antibodies). ${ }^{74}$

VD supplementation up to a dose of 10,000 IE/day must be given to achieve an individual, effective $25(\mathrm{OH}) \mathrm{D}$ level, checking VD serum levels and serum $\mathrm{Ca}$ levels. Uniform data on the VD daily dose for all PwMS are not possible because various specific genetic errors in the VD metabolism determine the serum VD level (eg, abnormalities of the gene of enzyme $1 \alpha$-hydroxylase CYP27B1 [cytochrome P 450 family 27 subfamily B member], SNPs [single-nucleotide polymorphisms] rs 703,842 or rs 10877,013). ${ }^{28}$ Another pathogenesis factor in the development of autoimmune disease is a VDR polymorphism. Genetic VD receptor mutations may result in an alteration of the effects produced by the binding of $1,25(\mathrm{OH}) 2 \mathrm{D}$ in the promoter regions of response to VD. The association of numerous SNPs and the risk of islet autoimmunity is increasingly confirmed. ${ }^{70}$ The determination of specific diabetes autoantibodies before the first AI infusion has the advantage of prematurely detecting high risk.

\section{"Surprises" in the repertoire of unwanted secondary autoimmune diseases \\ Lambert-Eaton myasthenic syndrome (LEMS)/myasthenia gravis}

New symptoms in PwMS can easily be misinterpreted when clinical signs are incorrectly attributed to the course of MS. Hoffman et al diagnosed LEMS after AI therapy. ${ }^{75}$ LEMS is caused by autoantibodies direct against calcium channels (anti-VGCC-AK) on the neuromuscular junction. IL-21 drives secondary autoimmunity in PwMS, following therapeutic lymphocyte depletion with AI. ${ }^{17}$ Jones et al suggest that serum IL-21 may, therefore, serve as a biomarker for the risk of developing autoimmunity month to years after AI treatment. Before treatment, PwMS who went on to develop secondary autoimmunity had more than twofold greater levels of serum IL-21 than the nonautoimmune group. ${ }^{17}$ Patients with myasthenia gravis had lower 25(OH)-plasma levels ${ }^{76}$ and by a "high-dose vitamin D treatment" in one case a complete remission could be achieved. However, the VD serum levels were in the extremely high range (400$700 \mathrm{ng} / \mathrm{mL}){ }^{77} \mathrm{VDS}(1,25(\mathrm{OH}) \mathrm{D})$ can attenuate the potential increase of IL-21 levels. Neurological autoimmune diseases as a side effect are a diagnostic pitfall.

\section{Autoimmune hepatitis}

The tendency to polyautoimmunity is complemented by publications on autoimmune hepatitis (AIH) in two cases after the second AI cycles and simultaneous manifestation of a Grave's disease. ${ }^{78,79}$ Canham et al diagnosed an autoimmune hepatitis in a 43-year-old Caucasian female after the second infusion-AI cycle 1 year later. ${ }^{80}$ There is an increase in the prevalence of AIH in PwMS compared to the general population $(0.17-1 \%$ vs $0.02 \%) .{ }^{81,82}$ In terms of differential diagnosis, AIH must be distinguished from a drug-induced liver injury caused by AI, which can be confirmed by re-exposure. ${ }^{83}$ As a supplement, liver injury after pulsed methylprednisolone therapy in PwMS with RRMS has been observed both as acute drug-induced liver injury and as $\mathrm{AIH}^{84,85} \mathrm{AIH}$ was observed in 40 reported cases in PwMS receiving disease-modifying drug therapy. ${ }^{86}$ However, patients with AIH have a high prevalence of VD deficiency. ${ }^{87}$ Low serum VD levels are associated with severe histological features and poor response to therapy in patients with autoimmune hepatitis. ${ }^{88}$ 1,25-dihydroxyvitamin D can inhibit immune cell proliferation, promote an antiinflammatory cytokine profil, expand regulatory T-cells, enhance glucocorticoid actions, increase glutathione production, and inhibit hepatic stellate cells. Loss of VDdependent homeostatic mechanism may promote disease progression. ${ }^{89}$ Severe VD deficiency is associated with treatment non-response, progression to cirrhosis, and liver-related death or need for liver transplant. Severe VD deficiency is a prognostic biomarker in AIH. ${ }^{9090}$ VD supplementation initially requires a higher VD dose (6,000-10,00 IU/day) to rapidly achieve an effective serum level (at least $30 \mathrm{ng} / \mathrm{mL}$ ), followed by a maintenance dose of 3,000-6,000 IU/day). These high doses are necessary because the AIH has excessive systemic inflammation at the time of diagnosis, supports obligatory 
glucocorticoid therapy and may have a "glucocorticoid saving effect". 90,91

The general importance of the daily dose of VD is pointed out by the study of $\mathrm{O}^{\prime}$ Connell et $\mathrm{al}^{92}$. The CISAVID trial ("Dose-related effects of vitamin D3 on immune responses in patients with clinically isolated syndrome" [NCT01728922; interventional, double-blind randomized placebo-controlled trial]) which also aimed to examine the immunologic effects of VD supplementation at two doses $(5,000 \mathrm{IU}$ or $10,000 \mathrm{IU} /$ day $)$ in patients with CIS over a 24-week treatment period. This study from Ireland has been completed, but no results of the study have been reported yet. ${ }^{93}$ The dose-response effect is decisive for the success of the therapy.

\section{Rare side effects - often a diagnostic puzzle?}

The importance of polyautoimmunity is demonstrated by the case report of the simultaneous occurrence of GD and acquired hemophilia A (AHA). AHA is extremely rare in patients treated with AI $\left(0.2 \%\right.$ in clinical trials) ${ }^{94}$ For bleeding, both the platelet count and the routine coagulations test (prothrombin time) and active partial thromboplastin time) should be included in the laboratory serological monitoring ${ }^{95,96}$ madeley of patients treated with AI.

In the presence of thyroid antibodies and manifestation of new neurological symptoms (eg, paresthesia, epilepsy) autoimmune encephalitis may rare develop. Giarola et al report a case of autoimmune encephalitis ("Hashimoto's Encephalopathy") manifesting as a polymorphic epilepsy partialis continua/status epilepticus 7 months after the second course of AI in a patient with previous autoimmune hypothyroidism and immune thrombocytopenic purpura. ${ }^{97}$ Metz et al report a case of disseminated necrotizing leukoencephalopathy and severe AIHA 8 months after AI treatment. ${ }^{98}$

\section{Vitiligo}

Ruck et al describe three cases of AI-treated patients with RRMS developing vitiligo 14, 18, 52 months after AI initiation (T-cell-mediated secondary autoimmune disease with increased IL-21).${ }^{99}$ Eichau et al diagnosed another case of vitiligo in a 28 -year-old female patient 5 months after the first AI cycle. ${ }^{100}$ Vitiligo and alopecia are both autoimmune diseases. VD represents a potential player in the pathogenesis of vitiligo. Low 25-hydroxyvitamin D levels are associated with vitiligo. ${ }^{101}$ IL-17 was significantly higher whereas VD was found to be lower among the patients. A significant positive correlation was noted between VD levels and disease duration. ${ }^{102}$ Zhou et al showed increased circulating Th17-cell frequencies and elevated serum IL17A, TGF- $\beta 1$, and IL-21 levels in patients with non-segmental vitiligo. ${ }^{103}$ In a pilot study, 16 patients with vitiligo received 35,000 IU VD/day for 6 months in combination with a low calcium diet. Avoidance of milk products and calcium-enriched foods as well as consumption of oats, rice, and soy milk is necessary. A fluid intake of at least $2.5 \mathrm{~L}$ per day had to be maintained. Fourteen of 16 vitiligopatients showed a repigmentation of $25-75 \%$. All patients presented low VD status (serum $25(\mathrm{OH}) \mathrm{D} \leq 30 \mathrm{ng} / \mathrm{mL}$ ). ${ }^{104}$

\section{Myalgia as an indication of secondary autoimmune myositis}

Aouad et al report on a first case of secondary myositis after AI therapy. Six months after the induction therapy with AI a 44-year-old patient with RRMS myalgia affecting both arms and legs in a predominantly proximal distribution developed. The blood test showed an extremely high level of creatine phosphokinase (CK) (>50-fold). The complete regression of the symptoms and the increased CK after oral glucocorticoid therapy speak for an autoimmune genesis. ${ }^{105}$ Azali et al could prove low serum levels of VD in idiopathic inflammatory myopathies (polymyositis, dermatomyositis, inclusion body myositis). ${ }^{106}$

Serum IL-17A level was high in patients with dermatomyositis and polymyositis and just as serum IL-6 and IFN $\gamma$ but also associated with disease activity. ${ }^{107}$ VDS is being considered as an adjuvant therapy. The supplementation of VD can also reduce the synthesis of inflammatory cytokines (IL-21, IL-17) and increase the synthesis of antiinflammatory cytokines.

\section{The knowledge of the tendency toward polyautoimmunity is the key to the exact interpretation of symptoms caused by unwanted side effects \\ Sarcoidosis}

Tyshkov et al confirm the trend toward polyautoimmunity through the report of comorbidity of sarcoidosis in 10 PwMS. ${ }^{108}$ Systematic sarcoidosis can either follow or precede the diagnosis of MS or occur simultaneously. ${ }^{109,110}$ In addition to these publications, Willis et al report on three cases of sarcoidosis after two Al cycles over a period of 2-4 years. These also showed comorbidity with hypothyroidism, GD, and ITP. ${ }^{111}$ Graf et al observed acute sarcoidosis 
(Löfgren's syndrome) 1.5 years after the second Al cycle and thus demonstrated the invasive effects of $\mathrm{Al}$ on the immune system. ${ }^{112}$ IFN- $\beta$-induced sarcoidosis has also been reported repeatedly. ${ }^{113}$ However, VD administration is not unproblematic. Up to $10 \%$ of cases of sarcoidosis develop hypercalcemia. ${ }^{114,115}$ In patients with sarcoidosis, hypercalcemia occurs as a result of the activity of ectopic 25(OH)D-1-hydroxylase (CYP27B1) expressed in macrophages. ${ }^{114,115}$ The constellation of elevated 1,25-dihydroxyvitamin $\mathrm{D}$ and low $25(\mathrm{OH}) \mathrm{D}$ serum values may be possible, the latter being used to control overdose. ${ }^{116}$ In $40-60 \%$, there is a hypercalciuria. ${ }^{117}$ There are controversial opinions on VD supplementation as add-on therapy for sarcoidosis. ${ }^{115}$ Miedema et al demonstrate a key role of Th17-cell plasticity in granuloma formation and maintenance. ${ }^{118}$ VD supplementation would affect the disease by suppressing the activity of Th-17cells and Kamphuis et al recommend supplementation because there was a significant negative correlation between $25(\mathrm{OH}) \mathrm{D}$ serum levels and disease activity. ${ }^{119}$ VD increases the therapeutic effects of glucocorticoids via an mTORc1-dependent upregulation of the glucocorticoid receptor. ${ }^{120}$ The risk of osteoporosis in the primary therapy of sarcoidosis with a glucocorticoid could be prevented by VD supplementation.

It is challenging to assign an absolute serum VD concentration over which toxicity is always present. ${ }^{103}$ However, serum total $25(\mathrm{OH}) \mathrm{D} 3$ concentrations $>80 \mathrm{ng} /$ $\mathrm{mL}(200 \mathrm{nmol} / \mathrm{L})$ should be a warning mark on monitoring, only concentrations typically severalfold higher than $80 \mathrm{ng} / \mathrm{mL}$ showed hypercalcemia. ${ }^{103}$ Monitoring by determination of serum calcium and phosphate is required.

A study on VD homeostasis in sarcoidosis is currently being conducted. This study evaluates the relationship between vitamin-D status and severity of sarcoidosis, and the effects of vitamin-D repletion in vitamin-D insufficient patients with sarcoidosis. ${ }^{121}$

\section{Conclusion}

Vitamin D, as an immune regulator, can modulate the immune system via endocrine, paracrine, and intracrine mechanisms. Extensive epidemiological research supports the causality of the link between low VD values and the occurrence and development of MS and autoimmune diseases. PwMS who developed autoimmune secondary diseases after AI treatment as side effects showed high basal levels of IL-21, a cytokine that increases the growth of autoreactive T- cells. Serum IL-21 levels may serve as biomarkers for the risk of developing autoimmunity months to years after treatment with AI. ${ }^{17} \mathrm{VD}$ is one of the factors that can regulate the function of Treg cells. Therapy with VD is inexpensive and without significant side effects. The mechanisms of polyautoimmunity with its "immunological labyrinth" are currently unclear, as are the exact physiological influences of VDS. However, randomized clinical trials testing VDS on polyautoimmunity are not possible due to virtually insurmountable practical difficulties.

Escasany's demands can only be supported by the fact that new side effects that have occurred in the meantime must be communicated to the doctors in good time, especially as not all side effects are published. Although lifethreatening adverse events are rare in long-term monitoring and there are no markers prior to alemtuzumab therapy to detect the possibility of secondary autoimmunity, ${ }^{122}$ interdisciplinary collaboration is the key to detecting rare adverse events.In the case of polyautoimmunity with rare combinations, e.g. MS and myasthenia gravis, a differential diagnostic distinction must be made between "natural occurrence of such coexistence" and secondary autoimmunity in the context of alemtuzumab treatment.

It is very likely that for this problem of side effects of AI therapy, no randomized clinical trials with VDS testing are possible in the short term. For side effects, such as thyroid dysfunction, autoimmune thrombocytopenia, AIHA, acquired hemophilia $\mathrm{A}$, diabetes mellitus type 1, autoimmune hepatitis, Lambert-Eaton myasthenic syndrome, sarcoidosis and AA/AT, vitiligo, or autoimmune myositis, VDS may have an impact on the immunological mechanism, in particular lowering levels of IL-17 and IL-21. The daily intake of $\mathrm{VD}$ and the attained $25(\mathrm{OH})$-serum level are decisive for the success of the therapy (dose-response effect). In daily practice, it should be noted that PwMS with oral VD supplementation has a lower increase in 25 $(\mathrm{OH}) \mathrm{D}$ serum levels (genetically caused hypovitaminosis D) than healthy individuals. The therapeutic potential of VD supplementation can only be used if regular monitoring of VD serum levels is performed to achieve individual optimal values (30 ng/mL [75 nmol/L] or higher). This control protects against rare intoxications observed at values $>150 \mathrm{ng} / \mathrm{mL}[375 \mathrm{nmol} / \mathrm{L}] .^{123}$ A prudent upper limit of $100 \mathrm{ng} / \mathrm{mL}(250 \mathrm{nmol} / \mathrm{L})$ for toxicity has a wide safety margin. ${ }^{124}$ The differential diagnosis of unexpected "atypical" symptoms in PwMS can be facilitated if doctors are sensitized to possible polyautoimmunity. Older age, females, higher education, obesity, and familial autoimmunity were significantly associated with polyautoimmunity. Ever smokers were marginally more likely to be 
polyautoimmune. Family autoimmunity may lead to earlier onset of MS. ${ }^{125}$

AEs under AI therapy as well as the tendency toward polyautoimmunity in PwMS require intensive interdisciplinary cooperation among physicians.

\section{Human and animal rights and informed consent}

This article does not contain any studies with human or animal subjects.

\section{Disclosure}

The author declares no conflicts of interest in this work.

\section{References}

1. Daniels GH, Vladic A, Brinar V, et al. Alemtuzumab-related thyroid dysfunction in a phase 2 trial of patients with relapsing-remitting multiple sclerosis. J Clin Endocrinol Metab. 2014;99(1):8090. doi:10.1210/jc.2013-2201

2. Baker D, Herrod SS, Alvarez-Gonzalez C, Giovannoni G, Schmierer K. Interpreting lymphocyte reconstitution data from the pivotal phase 3 trials of alemtuzumab therapy for multiple sclerosis. JAMA Neurol. 2017;74:907-908. doi:10.1001/ jamaneurol.2017.0325

3. Ranganathan U, Kaunzer U, Foster S, Vartanian T, Perumal JS. Immediate transient thrombocytopenia at the time of alemtuzumab infusion in multiple sclerosis. Mult Scler. 2018;24(4):540-542. doi: $10.1177 / 1352458517699876$

4. Puthenparampil M, Rinaldi F, Federle L, Cazzola C, Perini P, Gallo P. Decreased platelet number in multiple sclerosis during alemtuzumab infusion: a common, transient and clinical silent phenomenon. Ther $A d v$ Neurol Disord. 2018;11. Publ online. doi:10.1177/1756285617741056

5. Yap SM, McNicholas N, Hutchinson M, McGuigan C. Immediate thrombocytopenia at time of alemtuzumab infusion for multiple sclerosis - not always self-limiting, fully reversible or predictable. Mult Scler. 2018;24(4):552-553. doi:10.1177/1352458517719151

6. Meunier B, Rico A, Seguier J, et al. Life-threatening autoimmune warm hemolytic anemia following treatment for multiple sclerosis with alemtuzumab. Mult Scler. 2018;25:1-3. doi:10.1177/1352458517729766

7. Di Ioia M, Farina D, Di Tommaso V, et al. Simultaneous earlyonset severe autoimmune hemolytic anemia and albuminuria during alemtuzumab treatment for multiple sclerosis. Mult Scler. 2018;24:813-815. doi:10.1177/1352458517743093

8. Barcellini W. New insights in the pathogenesis of autoimmune hemolytic anemia. Trans Med Hemother. 2015;42(5):287-293. doi:10.1159/000439002

9. Perga S, Martire S, Montarolo F, et al. The footprints of polyautoimmunity: evidence for common biological factors involved in multiple sclerosis and hashimoto thyroiditis. Front Immunol. 2018;9:311. doi:10.3389/fimmu.2018.00311

10. Azzopardi L, Thompson SA, Harding KE, et al. Predicting autoimmunity after alemtuzumab treatment of multiple sclerosis. $J$ Neurol Neurosurg Psychiatry. 2014;85:795-798. doi:10.1136/ jnnp-2013-307042

11. Obermann M, Ruck T, Pfeuffer S, Baum J, Wiendl H, Meuth SG. Simultaneous early-onset immune thrombocytopenia and autoimmune thyroid disease following alemtuzumab treatment in relapsing-remitting- multiple sclerosis. Mult Scler. 2016;22(9):12351241. doi: $10.1177 / 1352458516638558$
12. Cuker A, Coles AJ, Sullivan H, et al. A distinctive form of immune thrombocytopenia in a phase 2 study of alemtuzumab for the treatment of relapsing-remitting multiple sclerosis. Blood. 2011;118(24):6299-6305. doi:10.1182/blood-2011-08-371138

13. Fattizzo B, Zaninoni A, Gianotta JA, Binda F, Cortelezzi A, Barcellini W. Reduced 25-OH vitamin D in patients with autoimmune cytopenias, clinical correlations and literature review. Autoimmun Rev. 2016;15:770-775. doi:10.1016/j.autrev.2016.03.015

14. Culic S, Markic J, Petrovic D, Konjevoda P, Pavelic J. Serum vitamin D levels in children with newly diagnosed and chronic immune thrombocytopenia. Semin Hematol. 2016;53(Suppl 1): S67-S69. doi:10.1053/j.seminhematol.2016.04.020

15. Zhang $\mathrm{Q}$, Bai $\mathrm{H}$, Wang $\mathrm{W}$. Increased percentages of $\mathrm{T}$ cells producing interleukn-21 in patients with immune thrombocytopenic purpura. Cell Biol Int. 2014;38(4):520-525. doi:10.1002/cbin.10220

16. Hu Y, Wang X, Yu S, Hou Y, Ma D, Hou M. Neutralizations of IL-17A and IL-21 regulate regulatory T cell/T-helper 17 imbalance via T-helper 17-associated signaling pathway in immune thrombocytopenia. Expert Opin Ther Targets. 2015;19(6):723732. doi:10.1517/14728222.2015.1016499

17. Jones JL, Phuah CL, Cox AL, et al. IL-21 drives secondary autoimmunity in patients with multiple sclerosis therapeutic lymphocyte depletion with alemtuzumab (Campath-1H). J Clin Invest. 2009;119(7):2052-2061. doi:10.1172/JCI37878

18. Aranow C. Vitamin D and the immune system. J Investig Med. 2011;59(6):881-886. doi:10.2310/JIM.0b013e31821b8755

19. Almerighi C, Sinistro A, Ciaprini C, Rocchi G, Bergamini A. 1Alpha,25-dihydroxyvitamin D3 inhibits CD40L-induced proinflammatory and immunomodulatory activity in human monocytes. Cytokine. 2009;45(3):190-197. doi:10.1016/j.cyto.2008.12.009

20. Jeffery LE, Burke F, Mura M, et al. 1,25-dihydroxyvitamin D3 and interleukin-2 combine to inhibit $\mathrm{T}$ cell production of inflammatory cytokines and promote development of regulatory $\mathrm{T}$ cells expressing CTLA-4 and FoxP3. J Immunol. 2009;183(9):5458-5467. doi:10.4049/ jimmunol.0803217

21. Kulling PM, Olson KC, Olson T, Feith DJ, Loughran T. Vitamin D in hematological disorders and malignancies. Eur J Haematol. 2017;98(3):187-197. doi:10.1111/ejh.12818

22. Bockow B, Bockow-Kaplan T. Refractory immune thrombocytopenia successfully treated with high-dose vitamin D supplementation and hydroxychloroquine: two case reports. J Med Case Rep. 2013;7:91. doi:10.1186/1752-1947-7-91

23. Schwalfenberg GK. Solar radiation and vitamin D: mitigating environmental factors in autoimmune disease. $J$ Environ Publ Health. 2012. doi:10.1155/2012/619381

24. Liu W, Li H, Hao Y, et al. Decreased immunosuppressive actions of 1Alpha-25-dihydroxyvitamin D3 in patients with immune thrombocytopenia. Mol Immunol. 2016;78:89-97. doi:10.1016/j.molimm.2016.08.014

25. Guo B. IL-10 modulates Th17 pathogenicity during autoimmune diseases. J Clin Cell Immunol. 2016;7(2):400. doi:10.4172/2155-9899

26. Ray A, Wang L, Dittel BN. IL-10-independent regulatory B-cell subsets and mechanisms of action. Int Immunol. 2015;27(10):531536. doi:10.1093/intimm/dxv033

27. Muraro PA, Scolding NJ, Fox RJ. Rare side effects of alemtuzumab remind us of the need for postmarketing surveillance. Neurology. 2018;90:819-820. doi:10.1212/WNL.0000000000005409

28. Pierrot-Deseilligny C, Souberbielle JC. Vitamin D and multiple sclerosis: an update. Mult Scler Relat Dis. 2017;14:35-45. doi:10.1016/j.msard.2017.03.014

29. Winter FD Jr. Immune thrombocytopenia associated with consumption of tonic water. Proc (Bayl Univ Med Cent). 2015;28(2):213216. doi:10.1080/08998280.2015.11929233

30. Rieckmann P, Lenz A, Hoffmann M, Poske U, Behr K, Kallmann B. Fatal autoimmune hemolytic anemia associated with alemtuzumab in a MS patient with severe relapsing remitting disease course and prior immune therapies . Neurology. 2016;86:P2.103. 
31. Reddel SW, Barnett MH, Riminton S, Dugal T, Buzzard K, Wang CT. Successful implementation of an automated electronic support system for patient safety monitoring: the alemtuzumab in multiple sclerosis safety systems (AMS3) study. Mult Scler. 2018; Epub ahead of print. doi: $10.1177 / 1352458518783673$

32. Saarela M, Senthil K, Jones J, et al. Hemophagocytic lymphohistiocytosis in 2 patients with multiple sclerosis treated with alemtuzumab. Neurology. 2018;90:849-851. doi:10.1212/WNL.0000000000005420

33. Croteau D, Flowers C, Kulick CG, et al. Acute acalculous cholecystitis: A new safety risk for patients with MS treated with alemtuzumab. Neurology. 2018;90:e1548-e1552. Epub ahead of print. doi:10.1212/WNL.0000000000005422

34. Zimmermann J, Buhl T, Müller M. Alopecia universalis following alemtuzumab treatment in multiple sclerosis: a barely recognized manifestation of secundary autoimmunity-report of a case and review of the literature. Front Neurol. 2017. doi:10.3389/ fneur.2017:00569

35. Leussink VI, Reifenberger J, Hartung HP. Case of alopecia universalis associated with alemtuzumab treatment in MS. Neurol Neuroimmunol Neuroinflamm. 2018;5:e454. doi:10.1212/ NXI.0000000000000454

36. Spano F, Donovan JC. Alopecia areata: part I: pathogenesis, diagnosis, and prognosis. Can Fam Physician. 2015;61:751-755.

37. Saif GAB. Severe subtype of alopecia areata is highly associated with thyroid autoimmunity. Saudi Med J. 2016;37:656-661. doi:10.15537/Smj.2016.6.13777

38. Goh C, Finkel M, Christos PJ, Sinha AA. Profile of 513 patients with alopecia areata: associations of disease subtypes with atopy, autoimmune disease and positive family history. $J$ Eur Acad Dermatol Venereol. 2006;20:1055-1060. doi:10.1111/j.14683083.2006.01676.x

39. Xing L, Dai Z, Jabbari A, et al. Alopecia areata is driven by cytotoxic T lymphocytes and is reversed by JAK inhibition. Nat Med. 2014;20(9):1043-1049. doi:10.1038/nm.3645

40. Wang ECE, Christiano AM. The changing landscape of alopecia areata: the translational landscape. Adv Ther. 2017;34(7):15861593. doi:10.1007/s12325-017-0540-9

41. Bhat YJ, Latif I, Malik R, et al. Vitamin D level in alopecia areata. Indian J Dermatol. 2017;62(4):407-410. doi:10.4103/ijd.IJD 67716

42. Lee S, Kim BJ, Lee CH, Lee WS. Increased prevalence of vitamin $\mathrm{D}$ deficiency in patients with alopecia areata: a systematic review and meta-analysis. J Eur Acad Dermatol Venereol. 2018;32 (7):1214-1221. doi:10.1111/jdv.14987

43. Bakry OA, Farargy SME, Shafiee MKE, Soliman A. Serum Vitamin D in patients with alopecia areata. Indian Dermatol Online J. 2016;7(5):371-377. doi:10.4103/2229-5178.190504

44. Chan JK, Traboulsee AL, Sayao AL. Case of alemtuzumab-related alopecia areata management in MS. Neurol Neuroimmunol Neuroinflamm. 2019;6(1):e516. doi:10.1212/NXI.0000000000000516

45. Tuohy O, Costelloe L, Hill-Cawthorne G, et al. Alemtuzumab treatment of multiple sclerosis: longterm safety and efficacy. $J$ Neurol Neurosurg Psychiatry. 2015;86:208-215. doi:10.1136/ jnnp-2014-307721

46. Cossburn M, Pace AA, Jones J, et al. Autoimmune disease after alemtuzumab treatment for multiple sclerosis in a multicenter cohort. Neurology. 2011;77:573-579. doi:10.1212/WNL.0b013e318228bec5

47. CAMMS223 Trial Investigators, Coles AJ, Compston DA, Selmaj $\mathrm{KW}$, et al. Alemtuzumab vs. interferon $\beta$-1a in early multiple sclerosis. N Engl J Med. 359;2008:1786-1801. doi:10.1056/NEJMoa0801936

48. Samuels MH. Subacute, silent, and postpartum thyroiditis. Med Clin North Am. 2012;96:223-233. doi:10.1016/j.mcna.2012.01.003

49. Rotondi M, Molteni M, Leporati P, Capelli V, Marino M, Chiovato L. Autoimmune thyroid diseases in patients treated with alemtuzumab for multiple sclerosis: an example of selective anti-TSHreceptor immune response. Front Endocrinol (Lausanne). 2017;8:254. doi:10.3389/fendo.2017.00254
50. Pariani N, Willis M, Muller I, et al. Alemtuzumab-induced thyroid dysfunction exhibits distinctive clinical and immunological features. J Clin Endocrinol Metab. 2018. Epub ahead of print. doi:10.1210/jc.2018-00359

51. Mahzari M, Arnaout A, Freedman MS. Alemtuzumab induced thyroid disease in multiple sclerosis: a review and approach to management. Can J Neurol Sci. 2015;42:284-291. doi:10.1017/ cjn. 2015.48

52. Bizzaro G, Shoenfeld Y. Vitamin D and autoimmune thyroid diseases: facts and unresolved questions. Immun Res. 2015;61:46-52. doi:10.1007/s12026-014-8579-Z

53. Ma J, Wu D, Li C, et al. Lower serum 25-hydroxyvitamin $\mathrm{D}$ level is associated with 3 types of autoimmune thyroid diseases. Medicine (Baltimore). 2015;94:e1639. doi:10.1097/MD.0000000000000874

54. Kivity S, Agmon-Levin N, Zisappl M, et al. Vitamin D and autoimmune thyroid disease. Cell Mol Immunol. 2011;8:243-247. doi:10.1038/cmi.2010.73

55. Tamer G, Arik S, Tamer I, Coksert D. Relative vitamin D insufficiency in Hashimoto's thyroiditis. Thyroid. 2011;21:891-896. doi:10.1089/thy.2009.0200

56. Mackawy AM, Al-Ayed BM, Al-Rashidi BM. Vitamin D deficiency and its association with thyroid disease. Int J Health Sci (Qassim). 2013;7:267-275. doi:10.12816/0006054

57. Wang X, Zynat J, Guo Y, et al. Low serum vitamin D is associated with anti-thyroid-globulin antibody in female individuals. Int $J$ Endocrinol. 2015;2015:1-6. doi:10.1155/2015/285290

58. Goswami R, Marwaha RK, Gupta N, et al. Prevalence of vitamin D deficiency and its relationships with thyroid autoimmunity in Asian Indians: a community-based survey. Br J Nutr. 2009;102:382-386. doi:10.1017/S0007114509220824

59. Chailurkit LO, Aekplakorn W, Ongphiphadhanakul B. High vitamin D status in younger individuals is associated with low circulation thyrotropin. Thyroid. 2013;23:25-30. doi:10.1089/ thy.2012.0001

60. Smolders J, Hupperts R, Vieth R, et al. High dose cholecalciferol (vitamin D3) oil as add-on therapy in subjects with RRMS receiving subcutaneous interferon B1a. 32nd Congress of the European Committee for Treatment and Research in Multiple Sclerosis; September 14-17, 2016; London. Free communications1; Abstract 166: 09-16-2016, ECTRIMS online library147013.

61. Baker D, Giovannoni G, Schmierer K. Marked Neutropenia: significant but rare in people with multiple sclerosis after alemtuzumab treatment. Mult Scler Relat Disord. 2017;18:181-183. doi:10.1016/j.msard.2017.09.028

62. Gaitán MI, Ysrraelit MC, Correale J. Neutropenia in patient with multiple sclerosis treated with alemtuzumab. JAMA Neurol. 2017;74(9):1143-1144. doi:10.1001/jamaneurol.2017.1456

63. Galgani S, Prosperini L, Haggiag S, Tortorella C, Gasperini C. Early transient asymptomatic neutropenia associated with alemtuzumab treatment in multiple sclerosis: a case report. $J$ Neurol. 2018;265:2152-2153. doi:10/1007/s00415-018-8976-7

64. Yiannopoulou KG, Papadimitriou D, Anastasiou AI, Siakantaris M. Neutropenia with fatal outcome in a multiple sclerosis patient 23 days after alemtuzumab infusion. Mult Scler Relat Disord. 2018;23:15-16. doi:10.1016/j.msard.2018.04.014

65. Willis MD, Harding KE, Pickersgill TP, et al. Alemtuzumab for multiple sclerosis: long term follow-up in an multi-centre cohort. Mult Scler. 2016;22(9):1215-1223. doi:10.1177/1352458515614092

66. Malmeström C, Andersson BA, Lycke J. First reports case of diabetes mellitus type 1 as a possible secondary autoimmune disease following alemtuzumab treatment in MS. $J$ Neurol. 2014;261:2016-2018. doi:10.1007/s00415-014-7448-y

67. Richter S, Wagner B, Gulowsden-Celius E. Two cases of diabetes mellitus type 1 after alemtuzumab treatment for multiple sclerosis another probable secondary autoimmune disease. J Neurology. 2019;266:1270-1271. doi:10.1007/s00415-019-09257-5 
68. Maddaloni E, Cavallari I, Napoli N, Conte C. Vitamin D and diabetes mellitus. Front Horm Res. 2018;50:161-176. doi: $10.1159 / 000486083$

69. Norris JM, Lee HS, Frederiksen B, et al. Plasma 25-hydroxyvitamin d concentrationen and risk of Islet autoimmunitiy. Diabetes. 2017. doi: $10.2337 / \mathrm{db} 17-0802$

70. Bizzaro G, Antico A, Fortunato A, Bizzaro N. Vitamin D and autoimmune diseases: is vitamin $\mathrm{D}$ receptor (VDR) polymorphismus the culprit? IMAJ. 2017;19:438-443.

71. Weydert JA. Vitamin D in children's health. Children (Basel). 2014;1(2):208-226. doi:10.3390/children 1020208

72. Hyppönen E, Läärä E, Reunanen A, Järvelin MR, Virtanen SM. Intake of Vitamin D and risk of type1 diabetes: a birth-cohort study. Lancet. 2001;358:1500-1503. doi:10.1016/S0140-6736(01)06580-1

73. Gorham ED, Garland CF, Burgi AA, et al. Lower prediagnostic serum 25-hydroxyvitamin D concentration is associated with higher risk of insulin-requiring diabetes: a nested case-control study. Diabetologia. 2012;55(12):3224-3227. doi:10.1007/s00125-012-2709-8

74. Talaat IM, Nasr A, Alsulaimani AA, et al. Association between type 1 , type 2 cytokines, diabetic autoantibodies and 25-hydroxyvitamin $\mathrm{d}$ in children with the type 1 diabetes. J Endocrinol Invest. 2016;39(12):1425-1434. doi:10.1007/s40618-016-0514-9

75. Hoffman BM, Zeid NA, Alam U, Caress JB. Lambert-Eaton myasthenic syndrom associated with alemtuzumab. Mult Scler Relat Disord. 2019;27:131-132. doi:10.1016/j.msard.2018.10.015

76. Kang SY, Kang JH, Choi JC, Song SK, Oh JH. Low serum vitamin D levels in patients with myasthenia gravis. J Clin Neurosci. 2018;50:294-297. doi:10.1016/j.jocn.2018.01.047

77. Cadegiani FA. Remission of severe myasthenia gravis after massive-dose vitamin D treatment. Am J Case Rep. 2016;17:51-54. doi:10.12659/ajcr.894849

78. Sankari E, Dahlqvist G, Laurent M, van Pesch V. Auto-immune hepatitis in a patients with multiple sclerosis treated with alemtuzumab. Acta Neurol Belg. 2018;118:331-333. doi:10.1007/s13760018-0922-Z

79. Carlson A, Bozinov N, Kipp L, Dunn J, Lock C. Autoimmune hepatitis during treatment of multiple sclerosis with alemtuzumab (P5.350). Neurology. 2018;90(15 Suppl.). doi:10.1212/ WNL.0000000000005560

80. Canham LJW, Mozayani B, Chelvaratnam U, et al. The first reported case of autoimmune hepatitis following alemtuzumab therapy for multiple sclerosis. ECTRIMS Online Library. 2017:199783. Abstract:ePoster1763.

81. De Seze J, Delcambre C, Fajardy I, et al. autoimmune hepatitis and multiple sclerosis: a coincidental association? Mult Scler. 2005;11:691-693. doi:10.1191/1352458505ms1239oa

82. Strassburg C, Beckebaum S, Geier A, et al. Practice guideline autoimmune liver diseases. Z Gastroenterol. 2017;55:1135-1226. doi:10.1055/s-0043-120199

83. Beattie W, Yan B, Sood S. Acute severe hepatitis with alemtuzumab and rechallenge after a year. J Clin Neurosci. 2019;60:158160. doi:10.1016/j.jocn.2018.10.055

84. Bresteacu C, Prevot S, Perlemuter G, Voican C. Methylprednisolone-induced acute liver injury in a patient treated for multiple sclerosis relapse. BMJ Case Rep. 2018;5. In Press. doi:10.1136/bcr-2017-223670

85. Nociti V, Biolato M, De Fino C, et al. Liver injury after pulsed methylprednisolone therapy in multiple sclerosis patients. Brain Behav. 2018;8(6):e00968. doi:10.1002/brb3.968

86. Cacao G, Santos E, Martins Silva A. Concurrent autoimmune hepatitis in multiple sclerosis. Mult Scler. 2018;24(3):350-353. doi: $10.1177 / 1352458517692885$

87. Beinhardt S, Eder M, Marculescu A, et al. High prevalence of vitamin $D$ deficiency in patients with autoimmune hepatitis: impact on clinical presentation and response to treatment. Z Gastroenterol. 2015;53:P61. doi:10.1055/s-0035-1551749
88. Efe C, Kav T, Aydin C, et al. Low serum vitamin D levels are associated with severe histological features and poor response to therapy in patients with autoimmune hepatitis. Dig Dis Sci. 2014;59 (12):3035-3042. doi:10.1007/s10620-014-3267-3

89. Czaja AJ, Montano-Loza AJ. Evolving role of vitamin D in immune-mediated disease and its implications in autoimmune hepatitis. Dig Dis Sci. 2019;64(2):324-344.

90. Ebadi M, Bhanji RA, Mazurak VC, Lytvyak E, Mason A, Czaja AJ. Severe vitamin D deficiency is a prognostic biomarker in autoimmune hepatitis. Aliment Pharmacol Ther. 2019;49(2):173-182. doi:10.1111/apt.15029

91. Imga NN, Efe C. Editorial: the role of vitamin D in autoimmune hepatitis. Aliment Pharmacol Ther. 2019;40:342. doi:10.1111/apt.15075

92. O'Connel K, Kelly S, Kinsella K, et al. Dose-related effects of vitamin $\mathrm{D}$ on immune responses in patients with clinically isolated syndrome and healthy control participants: study protocol for an exploratory randomized duble-blind- placebo-controlled trial. Trials. 2013;14:272. doi:10.1186/1745-6215-14-272

93. Breuer J, Loser K, Mykicki N, Wiendl H, Schwab N. Does the environment influence multiple sclerosis pathogenesis via UVB light and/or induction of vitamin D? J Neuroimmunol. 2018. doi:10.1016/j.jneuroim.2018.05.006

94. McCaughan G, Massey J, Sutton I, Curnow J. Acquired haemophilia A complicating alemtuzumab therapy for multiple sclerosis. BMJ Case Rep. 2017. doi:10.1136/bcr-2017-223016

95. Pisa M, Della Valle P, Coluccia A, et al. Acquired haemophilia A as a secondary auatoimmune disease after alemtuzumab treatment in multiple sclerosis: A case report. Mult Scler Rel Disord. 2019;27:403-405. doi:10.1016/j.msard.2018.11.029

96. Madeley J, Hodges G, Birchley A. Acquired haemophilia A as a secondary autoimmune disease after alemtuzumab treatment in multiple sclerosis: a case report. BMJ Case Rep. 2018. doi:10.1136/bcr-2018-226588

97. Giarola B, Massey J, Barnett Y, Rodrigues M, Sutton I. Autoimmune encephalitis following alemtuzumab treatment of multiple sclerosis. Mult Scler Relat Disord. 2019;28:31-33. doi:10.1016/j.msard.2018.12.004

98. Metz I, Rieckmann P, Kallmann B, Brück W. Disseminated necrotizing leukoencephalopathy eight month after alemtuzumab treatment for multiple sclerosis. Acta Neuropathol Commun. 2016;4:81. doi:10.1186/s40478-015-0253-8

99. Ruck T, Pfeuffer S, Schulte-Mecklenbeck A, et al. Vitiligo after alemtuzumab treatment. Secondary autoimmunity is not all about B cells. Neurology. 2018;91(24). doi:10.1212/WNL.0000000000005773

100. Eichau S, Lopez-Ruiz R, Ruiz-Pena JL, et al. Vitiligo with Koebner phenomenon in a patient with multiple sclerosis treated with alemtuzumab. Rev Neurol. 2018;66:395-396.

101. Upala S, Sanguankeo A. Low 25-hydroxyvitamin D levels are associated with vitiligo: a systematic review and meta-analysis. Photodermatol Photoimmunol Photomed. 2016;32(4):181-190. doi:10.1111/phpp.12241

102. Aly D, Mohammed F, Sayed K, et al. Is there a relation between vitamin D and interleukin-17 in Vitiligo? A cross-sectional study. Dermatology. 2017;233(6):413-418. doi:10.1159/000479925

103. Zhou L, Shi YL, Li K, et al. Increased circulating Th17 cells and elevated serum levels of TGF-beta and IL-21 are correlated with human non segmental vitiligo development. Pigment Cell Melanoma Res. 2015;28(3):324-329. doi:10.1111/pcmr.12355

104. Finamor DC, Coimbra RS, Neves LCM, et al. A pilot study assessing the effect of prolonged administration of high daily doses of vitamin on the clinical course of vitiligo and psoriasis. Dermatoendocrinol. 2013;5(1):222-234. doi:10.4161/derm.24808

105. Aoud P, Yiannakis C, Fernando SL, Parratt J. A case of autoimmune myositis after treatment with alemtuzumab for multiple sclerosis. Mult Scler Expert Translat Clinic. 2018. doi:10.1177/ 2055217318819012 
106. Azali P, Helmers B, Kockum I, et al. Low serum levels of vitamin D in idiopathic inflammatory myopathies. Ann Rheum Dis. 2013;72 (4):512-516. doi:10.1136/annrheumdis-2012-201849

107. Silva MG, Oba-Shinjo SM, Marie SKN, Shinjo SK. Serum interleukin-17A level is associated with disease activity of adult patients with dermatomyositis and polymyositis. Clin Exp Rheumatol. 2018. Epub ahead of print. PMID:30620283

108. Tyshkov C, Pawate S, Bradshaw MJ, et al. Multiple sclerosis and sarcoidosis. A case for coexistence. Neurol Clin Pract. 2019;9:218227. doi:10.1212/CPJ.0000000000000629

109. Reves-Mantilla M, Kimbrough DJ, Calabresi PA, Pardo-Villamizar CA. The systematic sarcoidosis and multiple sclerosis dilemma. ECTRIMS online-library.ectrims.congress.eu>ectrims; Berlin, Germany. October 12, 2018:228889

110. Kimbrough D, Reyes-Mantilla M, Jimenez Arango J, PardoVillamizar C. Identifying multiple sclerosis in patients with systemic sarcoidosis (P06.214). Neurology. 2013;80(7 Suppl) P06.214.

111. Willis MD, Hope-Gill B, Flood-Page P, et al. Sarcoidosis following alemtuzumab treatment for multiple sclerosis. Mult Scler. 2018;24 (13):1779-1782. doi:10.1177/1352458518790391

112. Graf J, Ringelstein M, Lepka K, et al. Acute sarkoidosis in a multiple sclerosis patient after alemtuzumab treatment. Mult Scler. 2018;24(13):1776-1778. doi:10.1177/1352458518771276

113. Carbonelli C, Montepietra S, Caruso A, et al. Monaldi. Sarcoidosis and multiple sclerosis: systemic toxicity associated with the use of interferon-beta therapy. Arch Chest Dis. 2012;77 (1):29-31.

114. Tebben PJ, Singh JR, Kumar R. Vitamin D-mediated hypercalcemia: mechnisms, diagnosis, and treatment. Endocr Rev. 2016;37 (5):521-547. doi:10.1210/er.2016-1070

115. Kempisty A, Lewandowska A, Kus J. Disturbances of calcium metabolism and vitamin D supplementation in sarcoidosis. Twoway street. Pol Merkur Lekarski. 2018;44(261):147-149.
116. Sodhi A, Aldrich T. Vitamin D supplementation: not so simple in sarcoidosis. Am J Med Sci. 2016;352(3):252-257. doi:10.1016/j. amjms.2016.05.027

117. Conron M, Young C, Beynon HL. Calcium metabolism in sarcoidosis and its clinical implications. Rheumatology (Oxford). 2000;39:707-713. doi:10.1093/rheumatology/39.7.707

118. Miedema JR, Kaiser Y, Broos CE, Wijsenbeek MS, Grundewald J, Kool M. Th17-lineage cells in pulmonary sarcoidosis and Löfgren's syndrome: friend or foe? J Autoimmune. 2018;87:8296. doi:10.1016/j.jaut.2017.12.012

119. Kamphuis LS, Bonte-Mineur F, van Laar JA, van Hagen PM, van Daele PL. Calcium and vitamin D in sarcoidosis: is supplementation safe? $J$ Bone Miner Res. 2014;29(11):2498-2503. doi:10.1002/jbmr.2262

120. Hoepner R, Bagnoud M, Pistor M, et al. Vitamin D increases glucocorticoid efficacy via inhibition of m TORC1 in experimental models of multiple sclerosis. Acta Neuropathol. 2019. doi:10.1007/ s00401-019-0218-8

121. U.S. National Library of Medicine. Vitamin D homeostasis in Sarcoidose. Available from: ClinicelTrials.gov. Identifer: NCT03621553.

122. Escasany AA. Reporting suspected adverse reactions to new commercialized medicines in real world. Mult Scler. 2018;24(6):816817. doi:10.1177/1352458518754999

123. Holick MF. Vitamin D deficiency. N Engl J Med. 2007;357:266281. doi:10.1056/NEJMra070553

124. Jones G. Pharmacokinetics of vitamin D toxicity. Am J Clin Nutr. 2008;88(2):582S-586S. doi:10.1093/ajcn/88.2.582S

125. Martyn-Dow B, Briggs FB. Characterizing polyautoimmunity and familial autoimmunity in a U.S. multiple sklerosis population. Mult Scler. 2019;25(1 Suppl.):20-156. ACTRIMS 05. 07.2019, biostatisticsP027. doi:10.1177/1352458519843086 


\section{Supplementary material}

Patient safety requires a rapid transfer of new findings into practice, which can currently take more than 15 years. Louis Pasteur already regretted more than 100 years ago that this delayed translation into practice is to the detriment of patients.

The famous French Scientist (1822-1895) once said: "To him who devotes his life to science, nothing can give more happiness than increasing the number of discoveries, but his cup of joy is full when the results of his studies immediately find practical applications".

\section{Reference}

1. Gaither CC, Cavazos-Gaither AE Gaither`s Dictionary of Scientific Quotations. Springer Science \& Business. Media 2008, page 361 .

\section{Publish your work in this journal}

Therapeutics and Clinical Risk Management is an international, peerreviewed journal of clinical therapeutics and risk management, focusing on concise rapid reporting of clinical studies in all therapeutic areas, outcomes, safety, and programs for the effective, safe, and sustained use of medicines. This journal is indexed on PubMed Central, CAS,
EMBase, Scopus and the Elsevier Bibliographic databases. The manuscript management system is completely online and includes a very quick and fair peer-review system, which is all easy to use. Visit http://www.dovepress.com/testimonials.php to read real quotes from published authors. 